\title{
Impact Evaluation of Noise Uncertainty in Spectrum Sensing under Middleton Class A Noise
}

\author{
Enwei Xu \\ School of Electrical and Information Engineering \\ Harbin Institute of Technology, Harbin, China 150001 \\ Email: xewmickey@gmail.com
}

\author{
Fabrice Labeau \\ Department of Electrical and Computer Engineering \\ McGill University, Montreal, Canada H3A 0G4 \\ Email: fabrice.labeau@mcgill.ca
}

\begin{abstract}
Reliable spectrum sensing is required to enable the effective use of Cognitive Radio (CR) networks. In practice, CRs not only have to cope with impulsive noises but also need to consider noise uncertainties. Small uncertainties of noise parameters are inevitable in any practical detector and the impact of these must be considered. This paper considers the energy detector based spectrum sensing under Middleton Class A noise with parameter uncertainties. Firstly, we show how analytical expressions of the probabilities of detection and false alarm can be derived. Secondly, we show that the mismatch of impulsive parameters $A$ and $\Gamma$ have little impact on performance and can thus generally be ignored but the uncertainty of noise power $\sigma_{Z}^{2}$ can induce $S N R$ Walls to the detector, which means that, no matter how many observations are obtained, the detector cannot robustly detect the primary signal. These results have implications for designing an enhanced energy detector for robust spectrum sensing.
\end{abstract}

Index Terms-Cognitive Radio, Spectrum Sensing, impulsive noise, noise uncertainties.

\section{INTRODUCTION}

In wireless communication systems, the need for higher data rates has increased due to multimedia applications. As a result, the limitation of the natural frequency spectrum has become more obvious. Cognitive Radio (CR) as a tempting solution to exploit the available spectrum has emerged. In order to make opportunistic usage of the frequency bands that are not heavily occupied by Primary Users (PU), the ability to measure or sense the absent of PU signal is necessary [1]. The task of obtaining awareness about the spectrum usage and the absence of primary users is called spectrum sensing. A number of different methods are proposed for identifying the presence of signal transmissions, such as energy-detector-based sensing [2], matched filtering based sensing [3], waveform-based sensing [4], cyclostationarity-based sensing [5] and radio identification based sensing [6] etc. Among these methods, energybased approaches are the most common because of their low computational and implementation complexities. In addition, energy-based detectors do not need any knowledge about the PU's signal.

This work was supported by Hydro-Quebec, the Natural Sciences and Engineering Research Council of Canada, and McGill University in the framework of the NSERC/Hydro-Quebec/McGill Industrial Research Chair in Interactive Information Infrastructure for the Power Grid.

The author Enwei Xu is funded by China Scholarship Council (CSC).
Most of the existing literature on spectrum sensing considers impairment by additive white Gaussian noise (AWGN) only. However, this assumption fails to model the behavior of certain noise types in practice, such as impulsive noise. The Middleton Class A noise model is one of the widely investigated statistical distributions that is used to model the man-made interference and the impulsive noise in different systems [7]. In energy-based sensing, the signal is detected by comparing the output of the energy detector with a threshold which depends on the noise floor. The threshold can be selected for finding an optimum balance between the probability of false alarm $P_{f_{a}}$ and the probability of detection $P_{d}$. In practice, the threshold is chosen to obtain a certain false alarm rate [8]. In AWGN scenario, the selection of the threshold is dependent, as we will show, on the noise variance; a small noise power estimation error can cause significant performance loss [9] [10]. Similarly, the selection of the threshold under Middleton Class A noise is dependent on the parameters of the noise model. The impact of the parameter mismatch on the sensing performance must be considered. However, none paper is dealing with this issue.

We consider the energy-detector based spectrum sensing under impulsive noise with noise uncertainty, providing the following contributions.

- We derive the analytical expressions of the probability of false alarm $P_{f_{a}}$ and the probability of detection $P_{d}$ when the PU signal is contaminated by Middleton Class A noise. In particular, we show the relationship between the observation samples required and the parameters of noise and the $S N R$ for achieving the target $P_{f_{a}}$ and $P_{d}$.

- We analyze the impact of the noise parameters uncertainty on sensing performance. We conclude that the uncertainty of impulsive parameters $A$ and $\Gamma$ have little impact on sensing performance and can thus generally be ignored, but the $S N R$ Wall phenomenon will arise with the uncertainty of noise power $\sigma_{Z}^{2}$.

The remainder of this paper is organized as follows. The signal model is defined in Section II. In Section III we give the derivation of the analytical expressions of the probability of false alarm and detection. We analyze the impact of the noise parameters uncertainty on sensing performance in Section IV and the conclusion is explained in Section V. 


\section{Signal Model}

In spectrum sensing problem, the PU signal to be sensed is considered as a random process (called Bayesian model) in some works, and it is also considered as an unknown deterministic signal (called classical model) in others [11]. Here we choose Bayesian model and consider a source $x$ with a zero-mean Gaussian PDF

$$
f_{X}(x)=G\left(x ; \sigma_{X}^{2}\right)=\frac{1}{\sqrt{2 \pi} \sigma_{X}} e^{-\frac{x^{2}}{2 \sigma_{X}^{2}}}
$$

transmitted over a channel impaired by a Middleton Class A noise $z$, whose PDF is

$$
f_{Z}(z)=\sum_{m=0}^{\infty} \beta_{m} G\left(z ; \sigma_{m}^{2}\right)=\sum_{m=0}^{\infty} \frac{\beta_{m}}{\sqrt{2 \pi} \sigma_{m}} e^{-\frac{z^{2}}{2 \sigma_{m}^{2}}}
$$

where $\beta_{m}=\frac{e^{-A} A^{m}}{m !}$ indicates that $m$ noise sources contribute to the impulsive event simultaneously, and $A=E\{m\}=$ $\sum_{m=0}^{\infty} m \beta_{m}$ is the corresponding overlap index denoting the average number of impulse noise sources active at any given time. Larger values of $A$ make the characteristic of the noise closer to Gaussian noise. Moreover, $\sigma_{Z}^{2}=E\left\{z^{2}\right\}=\sigma_{G}^{2}+$ $\sigma_{I}^{2}$ is the noise power, where $\sigma_{G}^{2}$ is the Gaussian power, $\sigma_{I}^{2}$ is the impulsive power. $\Gamma=\frac{\sigma_{G}^{2}}{\sigma_{I}^{2}}$ is the power-ratio of the Gaussian component to the impulsive component, and $\sigma_{m}^{2}=$ $\frac{\frac{m}{A}+\Gamma}{1+\Gamma} \sigma_{Z}^{2}=\sigma_{G}^{2}+\sigma_{I}^{2} \frac{m}{A}$. Thus, the Middleton Class A noise is totally characterized by the parameters $A, \Gamma$ and $\sigma_{Z}^{2}[12]$.

\section{Energy Detector Under Middleton Class A NOISE}

The problem of detecting the presence of primary users can be considered as the following binary hypothesis testing problem [13]:

$$
\begin{aligned}
& H_{0}: y(n)=z(n) \\
& H_{1}: y(n)=x(n)+z(n)
\end{aligned}
$$

where $n=1,2,3, \ldots, N, N$ is the number of observed samples; $y(n)$ is the signal observed by sensing receiver with $x(n)$ and $z(n)$ denoting the PU signal and the additive impulsive noise respectively. It is obvious that under hypothesis $H_{0}$, the PU signal is absent and $y(n)$ consists only of noise $z(n)$. On the contrary, under hypothesis $H_{1}$ the PU signal is present along with noise $z(n)$.

Assume the noise $z(n)$ and the signal $x(n)$ are independent of each other, we can obtain that

$$
\begin{aligned}
\left.f_{Y}(y(n))\right|_{H_{0}} & =f_{Z}(y(n))=\sum_{m=0}^{\infty} \frac{\beta_{m}}{\sqrt{2 \pi} \sigma_{m}} e^{-\frac{y(n)^{2}}{2 \sigma_{m}^{2}}} \\
\left.f_{Y}(y(n))\right|_{H_{1}} & =f_{X}(y(n)) * f_{Z}(y(n)) \\
& =\sum_{m=0}^{\infty} \frac{\beta_{m}}{\sqrt{2 \pi\left(\sigma_{m}^{2}+\sigma_{X}^{2}\right)}} e^{-\frac{y(n)^{2}}{2\left(\sigma_{m}^{2}+\sigma_{X}^{2}\right)}}
\end{aligned}
$$

As for the energy detector based spectrum sensing, the corresponding test statistic is expressed as:

$$
T(y)=\frac{1}{N} \sum_{n=1}^{N}|y(n)|^{2}
$$

According to the central limit theorem [14], when $N$ is large, the metric $T(y)$ can be approximated as a Gaussian random variable and

$$
\begin{aligned}
& \left.T(y)\right|_{H_{0}} \sim \mathcal{N}\left(\mu_{0}, \frac{\sigma_{0}^{2}}{N}\right) \\
& \left.T(y)\right|_{H_{1}} \sim \mathcal{N}\left(\mu_{1}, \frac{\sigma_{1}^{2}}{N}\right)
\end{aligned}
$$

in which

$$
\begin{aligned}
\mu_{0} & =\mathcal{E}\left\{|y(n)|^{2} \mid H_{0}\right\}=\sum_{m=0}^{\infty} \beta_{m} \sigma_{m}^{2}=\sigma_{Z}^{2} \\
\mu_{1} & =\mathcal{E}\left\{|y(n)|^{2} \mid H_{1}\right\} \\
& =\sum_{m=0}^{\infty} \beta_{m}\left(\sigma_{m}^{2}+\sigma_{X}^{2}\right)=\sigma_{Z}^{2}+\sigma_{X}^{2} \\
\sigma_{0}^{2} & =\mathcal{E}\left\{\left(|y(n)|^{2}-\mu_{0}\right)^{2} \mid H_{0}\right\} \\
& =\mathcal{E}\left\{|y(n)|^{4} \mid H_{0}\right\}-\mu_{0}^{2}=\sum_{m=0}^{\infty} 3 \beta_{m} \sigma_{m}^{4}-\sigma_{Z}^{4} \\
\sigma_{1}^{2} & =\mathcal{E}\left\{\left(|y(n)|^{2}-\mu_{1}\right)^{2} \mid H_{1}\right\} \\
& =\mathcal{E}\left\{|y(n)|^{4} \mid H_{1}\right\}-\mu_{1}^{2} \\
& =\sum_{m=0}^{\infty} 3 \beta_{m} \sigma_{m}^{4}-\sigma_{Z}^{4}+2 \sigma_{X}^{4}+4 \sigma_{X}^{2} \sigma_{Z}^{2}
\end{aligned}
$$

Thus, the probability of false alarm $P_{f_{a}}$ and probability of detection $P_{d}$ can be given in terms of the $Q$ function by

$$
\begin{gathered}
P_{f_{a}}=P_{r}\left(T(y)>\gamma \mid H_{0}\right)=Q\left(\frac{\gamma-\mu_{0}}{\sqrt{\sigma_{0}^{2} / N}}\right) \\
P_{d}=P_{r}\left(T(y)>\gamma \mid H_{1}\right)=Q\left(\frac{\gamma-\mu_{1}}{\sqrt{\sigma_{1}^{2} / N}}\right)
\end{gathered}
$$

where $\gamma$ is the threshold for the energy-based spectrum sensing.

If the variance of the signal $\sigma_{X}^{2}$ can be obtained in addition to the noise parameters, we can calculate $N$ by fixing the $P_{f_{a}}$ and $P_{d}$ as Eq.(9). Then the threshold $\gamma$ can also be obtained from $N$.

$$
N=\left[\frac{Q^{-1}\left(P_{f_{a}}\right) \sigma_{0}-Q^{-1}\left(P_{d}\right) \sigma_{1}}{\mu_{1}-\mu_{0}}\right]^{2}
$$

However, although the noise parameters can be estimated, the signal power is difficult to estimate since it depends on many varying factors such as transmission and propagation characteristics. In practice, the threshold is normally chosen to satisfy a certain $P_{f_{a}}$ according to the Neyman-Pearson criterion, which only requires the noise power to be known. 
As shown in Eq.(10):

$$
\gamma=\sqrt{\frac{\sigma_{0}^{2}}{N}} Q^{-1}\left(P_{f a}\right)+\mu_{0}
$$

\section{IMPACT EVALUATION OF UNCERTAINTY ON NOISE}

\section{CHARACTERISTICS}

Let $S N R=\frac{\sigma_{X}^{2}}{\sigma_{Z}^{2}}$, substituting into Eq.(9), we have

$$
N=\left[\frac{Q^{-1}\left(P_{f_{a}}\right) K(A, \Gamma)-Q^{-1}\left(P_{d}\right) \sqrt{K(A, \Gamma)^{2}+4 S N R+2 S N R^{2}}}{S N R}\right]^{2}
$$

where

$$
K(A, \Gamma)=\sqrt{\sum_{m=0}^{\infty} 3 \beta_{m}\left(\frac{m / A+\Gamma}{1+\Gamma}\right)^{2}-1}
$$

The Middleton Class A noise is totally characterized by the parameters $A, \Gamma$ and $\sigma_{Z}^{2}$. As in Eq.(11),when the three parameters are all known, $K(A, \Gamma)$ is determined. It is clearly that no matter how low the SNR is, the target $P_{f_{a}}$ and $P_{d}$ can be achieved by increasing the number of observation samples. However, the accurate noise parameter estimation is not always possible for the receiver [15].There must be small errors on the estimation of each parameter during the detecting process. So the impact of each of them must be considered. For discussion convenience, we assume without loss of generality, that the actual parameters are $A=0.01, \Gamma=0.1$ and $\sigma_{Z}^{2}=1$.

\section{A. Uncertainty of $A$}

With uncertainty, the estimated overlap index is assumed to be in an interval $\hat{A} \in\left[\frac{1}{\rho} A, \rho A\right]$, and $\rho>1$ is a parameter that quantifies the size of the uncertainty. To achieve a target $P_{f_{a}}$ and $P_{d}$ robustly, the following equations need to hold:

$$
\begin{aligned}
P_{f_{a}} & =\max _{\hat{A} \in\left[\frac{1}{\rho} A, \rho A\right]} Q\left(\frac{\gamma-\mu_{0}}{\sqrt{\sigma_{0}^{2} / N}}\right) \\
P_{d} & =\min _{\hat{A} \in\left[\frac{1}{\rho} A, \rho A\right]} Q\left(\frac{\gamma-\mu_{1}}{\sqrt{\sigma_{1}^{2} / N}}\right)
\end{aligned}
$$

Due to the fact that the $Q$ function is a monotonically decreasing function and $\sigma_{0}^{2}$ and $\sigma_{1}^{2}$ are also monotonically decreasing functions of $A$, we obtain

$$
\begin{array}{r}
P_{f_{a}}=\left.Q\left(\frac{\gamma-\mu_{0}}{\sqrt{\sigma_{0}^{2} / N}}\right)\right|_{\hat{A}=\frac{1}{\rho} A} . \\
P_{d}=\left.Q\left(\frac{\gamma-\mu_{1}}{\sqrt{\sigma_{1}^{2} / N}}\right)\right|_{\hat{A}=\rho A} .
\end{array}
$$

Then we have

$$
N=\left[\frac{Q^{-1}\left(P_{f_{a}}\right) K(A / \rho, \Gamma)-Q^{-1}\left(P_{d}\right) \sqrt{K(A \rho, \Gamma)^{2}+4 S N R+2 S N R^{2}}}{S N R}\right]^{2}
$$

Fig.1 shows the required number of samples $N$ to achieve the target $P_{f_{a}}=0.1$ and $P_{d}=0.9$ with different uncertainty levels $\rho$ on $A$. To achieve the target $P_{f_{a}}$ and $P_{d}$, a larger quantity of samples is required when the uncertainty of $A$ gets larger. Results for different $S N R$ is shown in Fig.2.

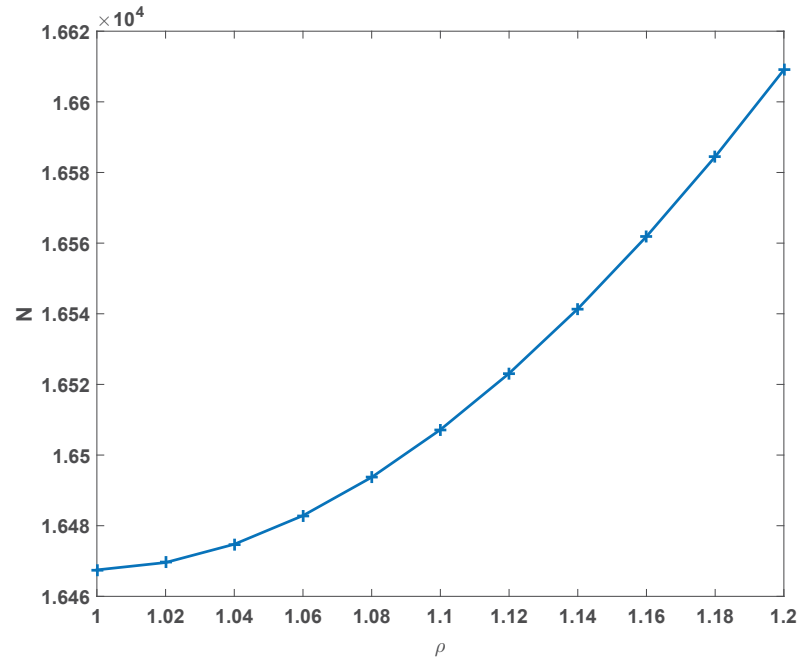

Fig. 1. Samples required vs. $\rho$, for different levels of uncertainty on $A .(\mathrm{SNR}=-5 \mathrm{~dB})$

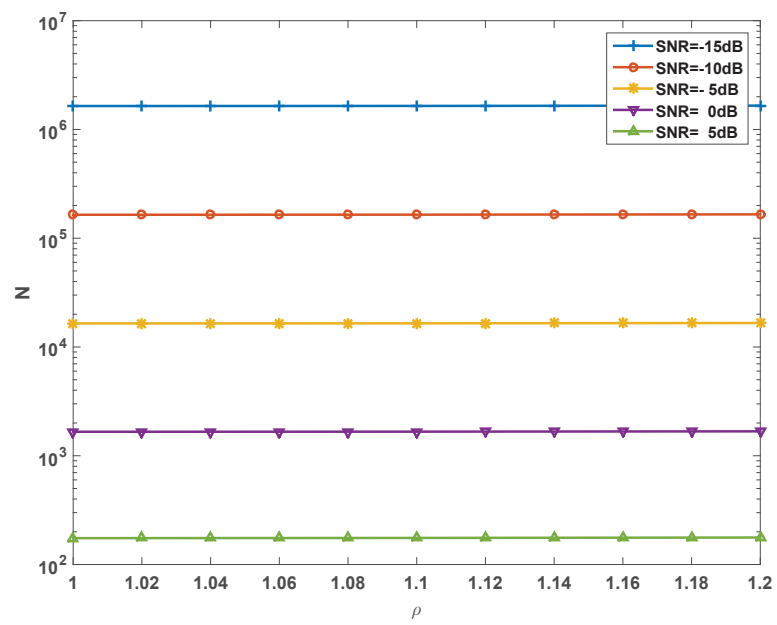

Fig. 2. Samples required vs. $\rho$, for different levels of uncertainty on $A$ with different $S N R$.

The two figures illustrate that we can slightly increase the number of samples $N$ to achieve the target $P_{f_{a}}$ and $P_{d}$. . The larger the uncertainty $\rho$ is, the larger $N$ is required. So the impact on the uncertainty of $A$ can be easily eliminated by increasing $N$, and $N$ is actually reasonably insensitive to $\rho$.

\section{B. Uncertainty of $\Gamma$}

Similarly, if the estimated power ratio is in an interval $\hat{\Gamma} \in$ $\left[\frac{1}{\rho} \Gamma, \rho \Gamma\right]$, and $\rho>1$ is a parameter that quantifies the size of the uncertainty, to achieve a target $P_{f_{a}}$ and $P_{d}$ robustly, the following equations need to hold:

$$
\begin{aligned}
P_{f_{a}} & =\max _{\hat{\Gamma} \in\left[\frac{1}{\rho} \Gamma, \rho \Gamma\right]} Q\left(\frac{\gamma-\mu_{0}}{\sqrt{\sigma_{0}^{2} / N}}\right) \\
P_{d} & =\min _{\hat{\Gamma} \in\left[\frac{1}{\rho} \Gamma, \rho \Gamma\right]} Q\left(\frac{\gamma-\mu_{1}}{\sqrt{\sigma_{1}^{2} / N}}\right) .
\end{aligned}
$$


With similar argument as above, we obtain

$$
\begin{array}{r}
P_{f_{a}}=\left.Q\left(\frac{\gamma-\mu_{0}}{\sqrt{\sigma_{0}^{2} / N}}\right)\right|_{\hat{\Gamma}=\frac{1}{\rho} \Gamma} . \\
P_{d}=\left.Q\left(\frac{\gamma-\mu_{1}}{\sqrt{\sigma_{1}^{2} / N}}\right)\right|_{\hat{\Gamma}=\rho \Gamma} .
\end{array}
$$

Then we have

$$
N=\left[\frac{Q^{-1}\left(P_{f_{a}}\right) K(A, \Gamma / \rho)-Q^{-1}\left(P_{d}\right) \sqrt{K(A, \Gamma \rho)^{2}+4 S N R+2 S N R^{2}}}{S N R}\right]^{2}
$$

Fig. 3 shows the required number of samples $N$ to achieve the target $P_{f_{a}}=0.1$ and $P_{d}=0.9$ with different uncertainty levels on $\Gamma$. To achieve the target $P_{f_{a}}$ and $P_{d}$, a smaller quantity of samples is required when the uncertainty of $\Gamma$ gets larger. Results for different $S N R$ is shown in Fig.4.

The two figures illustrate that we just need a smaller quantity of samples $N$ to achieve the target $P_{f_{a}}$ and $P_{d}$. The larger the uncertainty $\rho$ is, the smaller $N$ is required, but the variation in $N$ is very small. So the impact on the uncertainty of $\Gamma$ can in most cases be ignored.

Comparing the four figures, we found that the impact of $\Gamma$ is even lower than that of $A$, and the impact of these two parameters can be easily eliminated.

\section{Uncertainty of noise power $\sigma_{Z}^{2}$}

Finally, if the estimated noise power is assumed to be in an interval $\hat{\sigma}_{Z}^{2} \in\left[\frac{1}{\rho} \sigma_{Z}^{2}, \rho \sigma_{Z}^{2}\right]$, and $\rho>1$ is a parameter that quantifies the amount of the uncertainty. To achieve a target $P_{f_{a}}$ and $P_{d}$ robustly, the following equations need to hold:

$$
\begin{aligned}
& P_{f_{a}}=\max _{\hat{\sigma}_{z}^{2} \in\left[\frac{1}{\rho} \sigma_{z}^{2}, \rho \sigma_{z}^{2}\right]} Q\left(\frac{\gamma-\mu_{0}}{\sqrt{\sigma_{0}^{2} / N}}\right) \\
& P_{d}=\min _{\hat{\sigma}_{z}^{2} \in\left[\frac{1}{\rho} \sigma_{z}^{2}, \rho \sigma_{z}^{2}\right]} Q\left(\frac{\gamma-\mu_{1}}{\sqrt{\sigma_{1}^{2} / N}}\right) .
\end{aligned}
$$

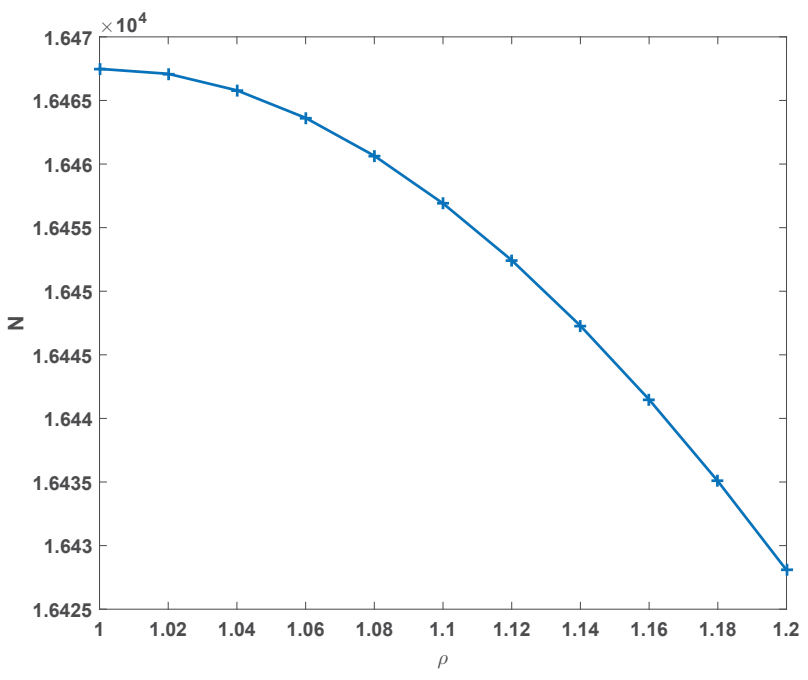

Fig. 3. Samples required vs. $\rho$, for different levels of uncertainty on $\Gamma$. $(\mathrm{SNR}=-5 \mathrm{~dB})$

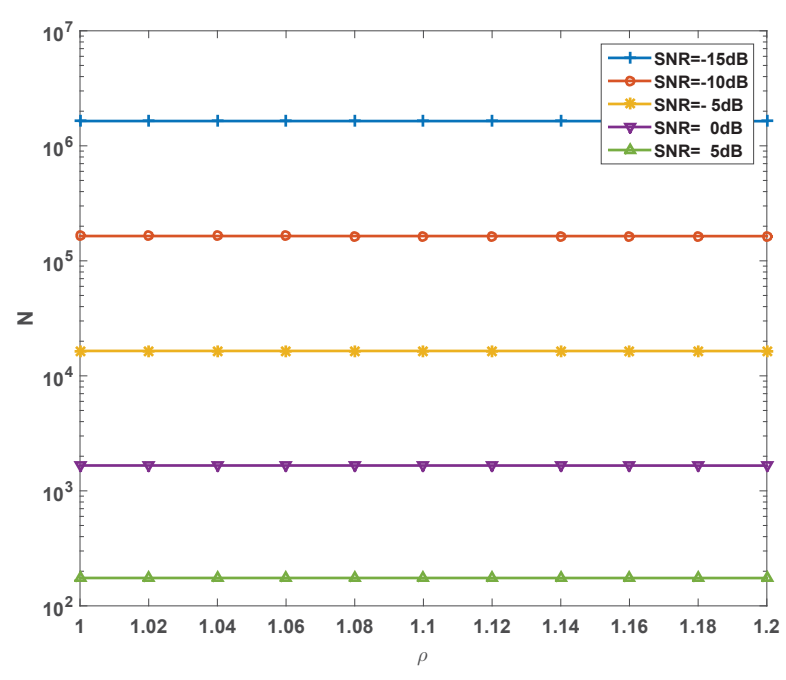

Fig. 4. Samples required vs. $\rho$, for different levels of uncertainty on $\Gamma$ with different $S N R$.

Due to the $Q$ function being a monotonically decreasing function, we obtain

$$
\begin{gathered}
P_{f_{a}}=\left.Q\left(\frac{\gamma-\mu_{0}}{\sqrt{\sigma_{0}^{2} / N}}\right)\right|_{\hat{\sigma}_{z}^{2}=\rho \sigma_{z}^{2}} \\
P_{d}=\left.Q\left(\frac{\gamma-\mu_{1}}{\sqrt{\sigma_{1}^{2} / N}}\right)\right|_{\hat{\sigma}_{z}^{2}=\frac{1}{\rho} \sigma_{z}^{2}}
\end{gathered}
$$

Then we have

$$
N=\left[\frac{Q^{-1}\left(P_{f_{a}}\right) K(A, \Gamma) \rho-Q^{-1}\left(P_{d}\right) \sqrt{K(A, \Gamma)^{2} \frac{1}{\rho^{2}}+\frac{4}{\rho} S N R+2 S N R^{2}}}{S N R-\left(\rho-\frac{1}{\rho}\right)}\right]^{2}
$$

It is obvious that, when $S N R$ approaches $\left(\rho-\frac{1}{\rho}\right), N \rightarrow \infty$. So an $S N R$ Wall exists. It means that the detector cannot robustly detect the signal if the signal power is less than the uncertainty in the noise power. Fig.5 shows that the $S N R$ wall will become higher with the uncertainty $\rho$ becoming higher.(note that the vertical axis is a logarithmic scale)

\section{CONCLUSION}

We studied the problem of detecting the presence of a primary user in a cognitive radio setting with impulsive noise. Firstly, we give the expression of false alarm probability $P_{f_{a}}$ and detection probability $P_{d}$ derived under the specific condition of impulsive communication noise. Secondly, we found that the uncertainty on noise parameters $A$ and $\Gamma$ have little impact on the detection performance, and the impact can be eliminated by adapting the number of samples $N$. Although both are small, uncertainty of A has larger impact on performance than that of $\Gamma$. Finally, the uncertainty of noise power $\sigma_{Z}^{2}$ cannot be ignored since it can result in the emergence of $S N R$ wall, in which case weak signals cannot be detected reliably no matter how many samples observed. 


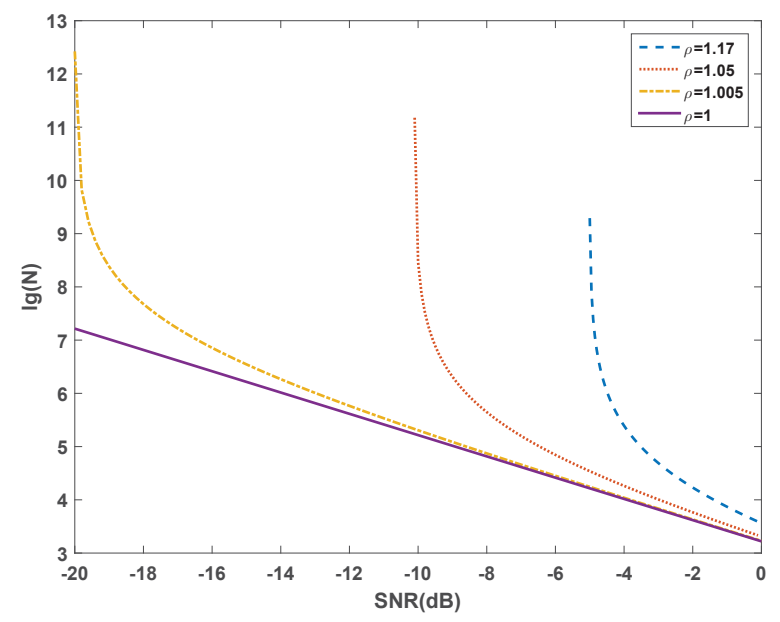

Fig. 5. SNR walls with different $\rho$.

\section{REFERENCE}

[1] T. Yucek and H. Arslan. A survey of spectrum sensing algorithms for cognitive radio applications. IEEE Communications SURVEYS Tutorials, 11(1):116-130, First 2009.

[2] Chang Liu, Ming Li, and Ming-Lu Jin. Blind energy-based detection for spatial spectrum sensing. IEEE Wireless Communications Letters, 4(1):98-101, Feb 2015.

[3] Zhang Xinzhi, Gao Feifei, Chai Rong, and Jiang Tao. Matched filter based spectrum sensing when primary user has multiple power levels. China Communications, 12(2):21-31, Feb 2015.

[4] M. Iqbal and A. Ghafoor. Analysis of multiband joint detection framework for waveform-based sensing in cognitive radios. In 2012 IEEE Vehicular Technology Conference (VTC Fall), pages 1-5, Sept 2012.

[5] G. Huang and J.K. Tugnait. On cyclostationarity based spectrum sensing under uncertain gaussian noise. IEEE Transactions on Signal Processing, 61(8):2042-2054, April 2013.

[6] W. Guibene, A. Hayar, M. Turki, and D. Slock. A complete framework for spectrum sensing based on spectrum change points detection for wideband signals. In 2012 IEEE 75th Vehicular Technology Conference (VTC Spring), pages 1-5, May 2012.

[7] D. Middleton. Non-gaussian noise models in signal processing for telecommunications: new methods an results for class a and class b noise models. IEEE Transactions on Information Theory, 45(4):11291149, May 1999.

[8] J.J. Lehtomaki, M. Juntti, H. Saarnisaari, and S. Koivu. Threshold setting strategies for a quantized total power radiometer. IEEE Signal Processing Letters, 12(11):796-799, Nov 2005.

[9] R. Tandra and A. Sahai. Snr walls for signal detection. IEEE Journal of Selected Topics in Signal Processing, 2(1):4-17, Feb 2008.

[10] A. Mariani, A. Giorgetti, and M. Chiani. Effects of noise power estimation on energy detection for cognitive radio applications. IEEE Transactions on Communications, 59(12):3410-3420, December 2011.

[11] Steven M. Kay. Fundamentals of Statistical Signal Processing: Estimation theory. Prentice-Hall Signal Processing Series, 1993.

[12] P. Banelli. Bayesian estimation of a gaussian source in middleton's classa impulsive noise. IEEE Signal Processing Letters, 20(10):956-959, Oct 2013.

[13] Harry Urkowitz. Energy detection of unknown deterministic signals. Proceedings of the IEEE, 55(4):523-531, April 1967.

[14] Athanasios Papoulis. Probability, Random Variables, and Stochastic Processes (3rd Edition). McGraw-Hill, 1991.

[15] L. Vijayandran, P. Dharmawansa, T. Ekman, and C. Tellambura. Analysis of aggregate interference and primary system performance in finite area cognitive radio networks. IEEE Transactions on Communications, 60(7):1811-1822, July 2012. 